\title{
Effect of alkyl chain length in the terminal ester group on mesomorphic properties of new chiral lactic acid derivatives
}

\author{
Michal Kohout*1, Alexej Bubnov ${ }^{2}$, Jiří Šturala ${ }^{1}$, Vladimíra Novotná ${ }^{2}$, and Jiří Svoboda ${ }^{1}$ \\ ${ }^{1}$ Department of Organic Chemistry, University of Chemistry and Technology, CZ-166 28 \\ Prague 6, Czech Republic \\ e-mail: Michal.Kohout@vscht.cz; Fax: +420220444182 \\ ${ }^{2}$ Institute of Physics, The Czech Academy of Sciences, Na Slovance 1999/2, 18221 Prague 8, \\ Czech Republic \\ e-mail: bubnov@fzu.cz; Fax:+420286890527
}

This supplementary electronic information summarizes results of quantum chemical calculations performed for materials of the series I and II.

The optimization of the structure was carried out at DFT level using M06L functional. [1] At first, we assumed that the energy barrier of the rotation of one dihedral angle is almost independent on the conformation of the others. This assumption allowed us to perform the relaxed scan optimization of the selected molecules with a 10 degrees step. We obtained the total energy of the molecules as a function of a dihedral angle using def2svp basis set with W06 density fitting basis set in vacuum. [2] By this approach, we determined the global minimum, other local minima and corresponding transition states connecting them. The final structures were further optimized with no constraints at M06L DFT level with def2tzvp basis set and W06 density fitting basis set, and at B3LYP DFT level [3] with def2tzvp basis set.

The energetic minima corresponding to the particular dihedral angle had the same order at both DFT functionals, but corresponding transition states connecting them were different. All minima had no imaginary frequency, while the transition states had the only one imaginary frequency corresponding to rotation, which was confirmed by frequency calculation at the same level of theory. All local minima differed from the global minimum by no more than $2.5 \mathrm{~kJ} / \mathrm{mol}$ and transition states from the global minimum by no more than 32.1 $\mathrm{kJ} / \mathrm{mol}$ (which does not need to correspond to the real barrier).

In accordance with the previously reported data, [4] a carbonyl group connected to an aromatic unit lies in the plane of the respective aromatic ring, while the second aromatic unit 
connected to the ester oxygen atom is rotated to another plane. [4] Such situation is in case of our materials represented by the dihedral angle $\chi$ (Figure ESI 1). The mutual rotation of the connected units lowers steric hindrance between the carbonyl unit and hydrogens of the neighbouring aromatic unit. Conversely, there is no steric hindrance for simple alkyl chains, thus, the alkyl chain of the biphenyl ester unit lies within the plane of its neighbouring aromatic ring (dihedral angle $\varepsilon$ ). The rotation of the lactic part (dihedral angle $\alpha$ ) generates a polarization vector and therefore can be considered as a constant for all studied materials. The major contribution to the free energy difference thus depends on the mutual orientation of benzene rings in the biphenyl part (dihedral angle $\delta$ ), and the orientation of moieties attached to the chiral lactic acid unit (dihedral angle $\beta, \chi$ ).<smiles>[X]c1ccc(-c2ccc(C(=O)OC)cc2)cc1</smiles>

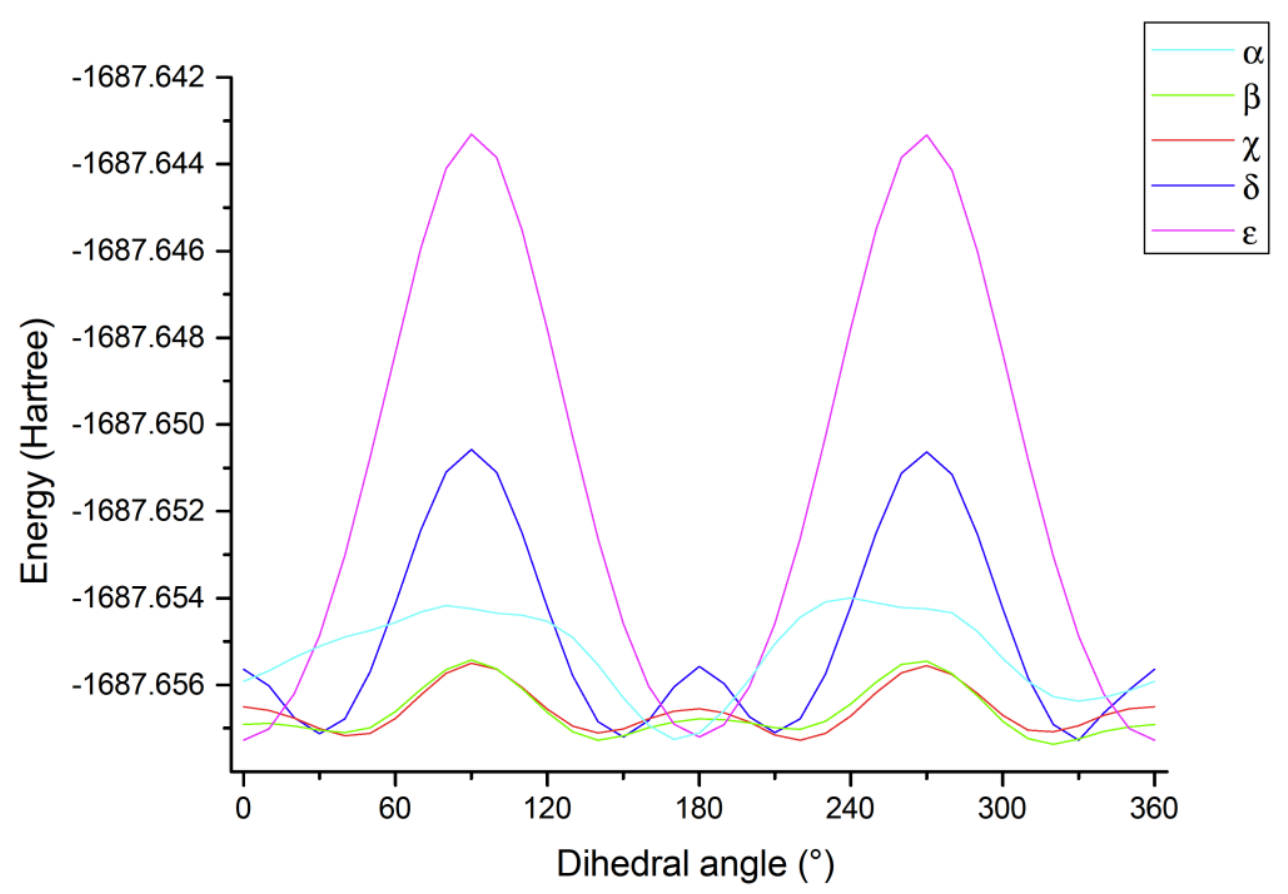

Figure ESI 1 Dependence of total energy on dihedral angles for compound Ia. 


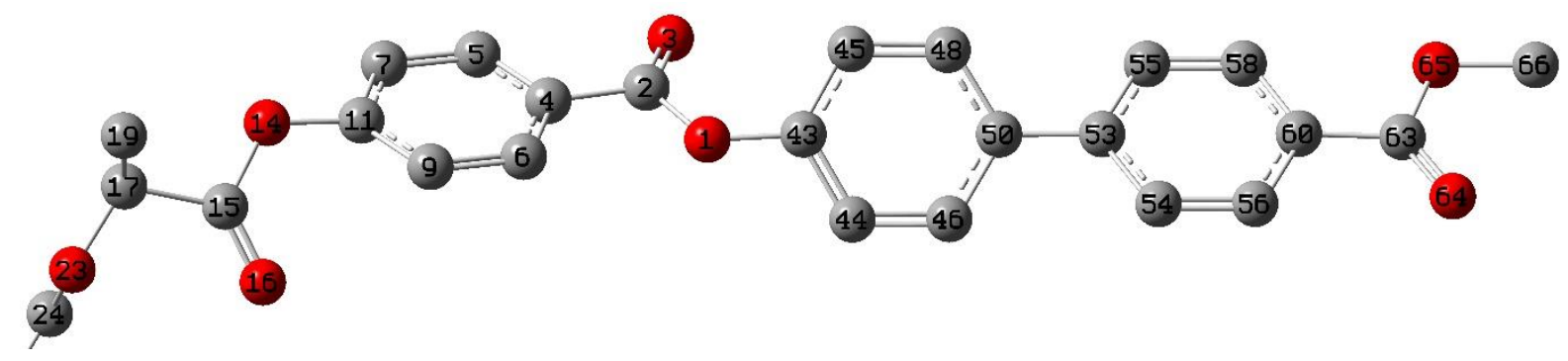

Figure ESI 2 Optimized structure of the material Ia. All hydrogen atoms and the terminal hexyl chain of the lactic acid unit are removed for clarity.

\section{Table ESI 1}

Summary of absolute and relative energies in respective minima and transition states for indicated dihedral angles for the compound Ia using different calculation functionals.

\section{Dihedral Structure
angle \\ global_minimum \\ ArCoo_min 140

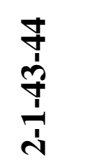 \\ ArCoo_min320 \\ ArCoo_min40 \\ ArCoo_ts0 \\ ArCoo_ts 180 \\ ArCoo_ts 270 \\ ArCoo_ts90}

\begin{tabular}{|c|c|}
\hline \multirow{7}{*}{ 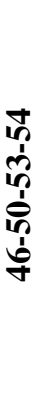 } & $\begin{array}{l}\text { global_minimum } \\
\text { biphen_min } 150\end{array}$ \\
\hline & biphen_min210 \\
\hline & biphen_min30 \\
\hline & biphen_ts0 \\
\hline & biphen_ts 180 \\
\hline & biphen_ts 270 \\
\hline & biphen_ts90 \\
\hline
\end{tabular}

$\begin{array}{rr}-1689.48294107 & 0.00 \\ -1689.48291832 & 0.06 \\ -1689.48280945 & 0.35 \\ -1689.48280352 & 0.36 \\ -1689.48111016 & 4.81 \\ -1689.48109457 & 4.85 \\ -1689.47624076 & 17.59 \\ -1689.47619728 & 17.71\end{array}$

$-1689.48294107$

$-1689.48284230$

$-1689.48263216$

$-1689.48271991$

$-1689.48187562$

$-1689.48175585$

0.00

0.26

0.81

0.58

2.80

3.11
M06L/def2tzvp

$\begin{array}{crr}\begin{array}{c}\text { Energy } \\ \text { [Hartree/mol] }\end{array} & \begin{array}{c}\Delta \mathbf{E} \\ {[\mathbf{k J} / \mathbf{m o l}]}\end{array} & \begin{array}{r}\text { Dihedral_angle } \\ {\left[{ }^{\circ}\right]}\end{array} \\ -1689.48294107 & 0.00 & -136.25 \\ -1689.48283754 & 0.27 & 136.51 \\ -1689.48282595 & 0.30 & -47.81 \\ -1689.48291832 & 0.06 & 47.35 \\ -1689.48171928 & 3.21 & -0.75 \\ -1689.48174520 & 3.14 & 179.12 \\ -1689.48168721 & 3.29 & -91.01 \\ -1689.48165957 & 3.36 & 91.24\end{array}$

-30.72
149.03
-148.65
30.80
0.382
-179.62
-90.30
89.85

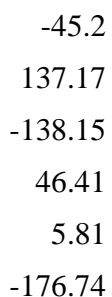

B3LYP/def2tzvp

$\begin{array}{crr}\begin{array}{c}\text { Energy } \\ {[\mathbf{H a r t r e e} / \mathbf{m o l}]}\end{array} & \begin{array}{c}\Delta \mathbf{E} \\ {[\mathbf{k J} / \mathbf{m o l}]}\end{array} & \begin{array}{r}\text { Dihedral_angle } \\ {\left[{ }^{\circ}\right]}\end{array} \\ -1689.73209325 & 0.00 & -126.27 \\ -1689.73200538 & 0.23 & 125.60 \\ -1689.73199632 & 0.25 & -59.09 \\ -1689.73205393 & 0.10 & 57.68 \\ -1689.73088094 & 3.18 & -0.85 \\ -1689.73093886 & 3.03 & 178.81 \\ -1689.73185866 & 0.62 & -90.02 \\ -1689.73184077 & 0.66 & 91.66\end{array}$

$\begin{array}{ll}-1689.73209325 & 0.00 \\ -1689.73206881 & 0.06 \\ -1689.73201861 & 0.20 \\ -1689.73201242 & 0.21 \\ -1689.72928292 & 7.38 \\ -1689.72924639 & 7.47 \\ -1689.72843895 & 9.59 \\ -1689.72840670 & 9.68\end{array}$

$-37.87$

142.17

$-141.87$

38.01

0.24

$-179.81$

$-90.25$

89.86 
PhcooLac_ts270

PhcooLac_ts90
$-1689.48147065$

$-1689.48148907$
3.86

3.81
$-92.65$

91.32

$-0.02$

179.96

$-91.14$

90.86

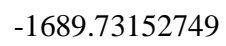

1.49

$-1689.73147726$
$-97.47$

89.32

$-0.01$

$-179.91$

$-91.22$

90.93

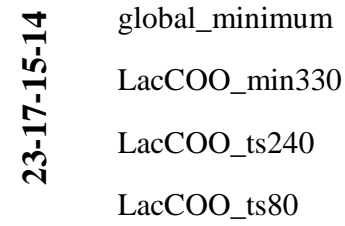

$\begin{array}{ll}-1689.48294107 & 0.00 \\ -1689.48222872 & 1.87 \\ -1689.47928575 & 9.60 \\ -1689.47966580 & 8.60\end{array}$

168.60
-31.70
-123.4
75.49

$-1689.73209325$

0.00

165.13

$-1689.73119728$

2.35

$-30.49$

$-1689.72828385$

10.00

$-126.15$

$-1689.72859589$

9.18

86.33

The global minima and transition states for the rest of the studied materials differed only in the total energy of the molecule.

\section{Table ESI 2}

The xyz coordinates of respective atoms of compound Ia in the global minimum calculated with B3LYP and M06L functional, respectively.

b3lyp_globmin

$\begin{array}{lrrr}\mathrm{O} & 1.36708200 & -0.72069400 & -0.14976000 \\ \mathrm{C} & 0.69911700 & -1.16632900 & 0.95776800 \\ \mathrm{O} & 1.22471300 & -1.34219500 & 2.02440300 \\ \mathrm{C} & -0.74031300 & -1.39297300 & 0.67252200 \\ \mathrm{C} & -1.55455900 & -1.81434700 & 1.72576600 \\ \mathrm{C} & -1.29957900 & -1.20798600 & -0.59387000 \\ \mathrm{C} & -2.90419900 & -2.04149200 & 1.51915500 \\ \mathrm{H} & -1.11362500 & -1.95815400 & 2.70248000 \\ \mathrm{C} & -2.64971400 & -1.43712300 & -0.81094900 \\ \mathrm{H} & -0.67463300 & -0.88522500 & -1.41359300 \\ \mathrm{C} & -3.44313500 & -1.84984900 & 0.25269500 \\ \mathrm{H} & -3.54736600 & -2.37026700 & 2.32420700 \\ \mathrm{H} & -3.08058400 & -1.28912800 & -1.78943500 \\ \mathrm{O} & -4.78529400 & -2.17077800 & 0.09963800 \\ \mathrm{C} & -5.64947700 & -1.33471600 & -0.56186800 \\ \mathrm{O} & -5.35134300 & -0.26449700 & -0.99997800 \\ \mathrm{C} & -7.02660500 & -2.00338500 & -0.64565900 \\ \mathrm{H} & -7.23207400 & -2.44200100 & 0.34084400 \\ \mathrm{C} & -7.00466800 & -3.10988800 & -1.69821100 \\ \mathrm{H} & -7.97932900 & -3.59596400 & -1.72474200 \\ \mathrm{H} & -6.80450000 & -2.68396500 & -2.68207700 \\ \mathrm{H} & -6.24344000 & -3.85406200 & -1.46693100 \\ \mathrm{O} & -8.02530300 & -1.08875600 & -1.00116400 \\ \mathrm{C} & -8.39090100 & -0.15982800 & 0.01687800 \\ \mathrm{C} & -9.51568900 & 0.71263600 & -0.50751500 \\ \mathrm{H} & -7.52801900 & 0.45699700 & 0.29169500 \\ \mathrm{H} & -8.71455200 & -0.71068200 & 0.91198900 \\ \mathrm{C} & -9.98970300 & 1.74267200 & 0.51822600 \\ \mathrm{H} & -9.16797300 & 1.21837400 & -1.41261500\end{array}$




$\begin{array}{lccc}\mathrm{H} & -10.34918900 & 0.07002900 & -0.80633800 \\ \mathrm{H} & -10.32307400 & 1.22842100 & 1.42706900 \\ \mathrm{H} & -9.14584800 & 2.37411000 & 0.81819400 \\ \mathrm{C} & -11.12183000 & 2.63102100 & 0.00163000 \\ \mathrm{C} & -11.60316000 & 3.66451700 & 1.02025300 \\ \mathrm{H} & -11.96664800 & 2.00121100 & -0.30011100 \\ \mathrm{H} & -10.78994900 & 3.14686200 & -0.90658600 \\ \mathrm{C} & -12.73400800 & 4.54762200 & 0.49626900 \\ \mathrm{H} & -11.93432700 & 3.14854900 & 1.92789500 \\ \mathrm{H} & -10.75876200 & 4.29382000 & 1.32100600 \\ \mathrm{H} & -13.05408500 & 5.27318800 & 1.24668500 \\ \mathrm{H} & -12.42165200 & 5.10406800 & -0.39098300 \\ \mathrm{H} & -13.60617400 & 3.94983700 & 0.21967400 \\ \mathrm{C} & 2.73525800 & -0.47080000 & -0.08775900 \\ \mathrm{C} & 3.16920400 & 0.78145700 & -0.49426800 \\ \mathrm{C} & 3.64485100 & -1.45188800 & 0.28002400 \\ \mathrm{C} & 4.52871000 & 1.05541900 & -0.53210100 \\ \mathrm{H} & 2.44128700 & 1.53201100 & -0.77224500 \\ \mathrm{C} & 5.00064000 & -1.16310400 & 0.24498100 \\ \mathrm{H} & 3.29852400 & -2.42731500 & 0.58925300 \\ \mathrm{C} & 5.47161900 & 0.09138200 & -0.16033700 \\ \mathrm{H} & 4.85825700 & 2.04250600 & -0.82884200 \\ \mathrm{H} & 5.70693200 & -1.93789900 & 0.51324300 \\ \mathrm{C} & 6.92144200 & 0.38719800 & -0.19532600 \\ \mathrm{C} & 7.47447600 & 1.17063700 & -1.21679500 \\ \mathrm{C} & 7.78205700 & -0.10727700 & 0.79252700 \\ \mathrm{C} & 8.83022300 & 1.44773200 & -1.24961100 \\ \mathrm{H} & 6.83730500 & 1.54475800 & -2.00761700 \\ \mathrm{C} & 9.13989200 & 0.16761600 & 0.76379100 \\ \mathrm{H} & 7.37775600 & -0.69275100 & 1.60806400 \\ \mathrm{C} & 9.67850600 & 0.94935900 & -0.25969400 \\ \mathrm{H} & 9.25411900 & 2.04568200 & -2.04496500 \\ \mathrm{H} & 9.78584900 & -0.21583100 & 1.54053700 \\ \mathrm{C} & 11.12638600 & 1.27754000 & -0.34344400 \\ \mathrm{O} & 11.62631900 & 1.94996100 & -1.21392200 \\ \mathrm{O} & 11.83913600 & 0.73946300 & 0.66824200 \\ \mathrm{C} & 13.24695100 & 1.01501100 & 0.65469600 \\ \mathrm{H} & 13.42726900 & 2.08794300 & 0.71333400 \\ \mathrm{H} & 13.70362500 & 0.62841600 & -0.25587600 \\ & 13.65165100 & 0.51142800 & 1.52847800\end{array}$

m061_globmin

$\begin{array}{lccc}\mathrm{O} & -1.30317100 & -0.70604500 & 0.12471900 \\ \mathrm{C} & -0.67165400 & -1.32117800 & -0.92095000 \\ \mathrm{O} & -1.22157800 & -1.64034000 & -1.94062100 \\ \mathrm{C} & 0.76026700 & -1.52089300 & -0.63199300 \\ \mathrm{C} & 1.54663300 & -2.10021300 & -1.62639100 \\ \mathrm{C} & 1.34682700 & -1.16370800 & 0.58103100 \\ \mathrm{C} & 2.89372000 & -2.31120300 & -1.41729700 \\ \mathrm{H} & 1.07826800 & -2.37689000 & -2.56154700 \\ \mathrm{C} & 2.69485300 & -1.37375500 & 0.80256800 \\ \mathrm{H} & 0.73845200 & -0.72022600 & 1.35630900 \\ \mathrm{C} & 3.46028500 & -1.94349900 & -0.20528200 \\ \mathrm{H} & 3.51909700 & -2.75983800 & -2.17684400 \\ \mathrm{H} & 3.14970600 & -1.09697200 & 1.74125300 \\ \mathrm{O} & 4.79722800 & -2.24866700 & -0.04004400 \\ \mathrm{C} & 5.64923600 & -1.35176000 & 0.54912700 \\ \mathrm{O} & 5.34596500 & -0.24859300 & 0.88987200 \\ \mathrm{C} & 7.01950600 & -2.00048900 & 0.69258100 \\ \mathrm{H} & 7.25926300 & -2.46354000 & -0.27776900\end{array}$




\begin{tabular}{|c|c|c|c|}
\hline $\mathrm{C}$ & 6.98191200 & -3.06880100 & 1.76721400 \\
\hline $\mathrm{H}$ & 7.95728200 & -3.54246100 & 1.84591500 \\
\hline $\mathrm{H}$ & 6.74248200 & -2.62284400 & 2.73131100 \\
\hline $\mathrm{H}$ & 6.23960000 & -3.82893900 & 1.53666900 \\
\hline $\mathrm{O}$ & 7.98927800 & -1.06161400 & 1.04631800 \\
\hline $\mathrm{C}$ & 8.33519100 & -0.16890600 & 0.00056600 \\
\hline $\mathrm{C}$ & 9.37351200 & 0.79559400 & 0.51106300 \\
\hline $\mathrm{H}$ & 7.44732600 & 0.37468400 & -0.34614500 \\
\hline $\mathrm{H}$ & 8.72093100 & -0.74146500 & -0.85697000 \\
\hline $\mathrm{C}$ & 9.81562400 & 1.78987100 & -0.54679600 \\
\hline $\mathrm{H}$ & 8.96414000 & 1.32369800 & 1.37575800 \\
\hline $\mathrm{H}$ & 10.23348600 & 0.23110300 & 0.88106300 \\
\hline $\mathrm{H}$ & 10.21248900 & 1.25202400 & -1.41449800 \\
\hline $\mathrm{H}$ & 8.94457800 & 2.33927300 & -0.91891300 \\
\hline $\mathrm{C}$ & 10.85608200 & 2.77647600 & -0.04926900 \\
\hline $\mathrm{C}$ & 11.29872100 & 3.77650900 & -1.10261800 \\
\hline $\mathrm{H}$ & 11.72891100 & 2.22915500 & 0.32237400 \\
\hline $\mathrm{H}$ & 10.46092800 & 3.31385100 & 0.81922800 \\
\hline $\mathrm{C}$ & 12.33594500 & 4.75803100 & -0.59658800 \\
\hline $\mathrm{H}$ & 11.69170700 & 3.23591000 & -1.96886200 \\
\hline $\mathrm{H}$ & 10.42379800 & 4.31964700 & -1.47191800 \\
\hline $\mathrm{H}$ & 12.63803400 & 5.46558400 & -1.36730500 \\
\hline $\mathrm{H}$ & 11.95599500 & 5.33567700 & 0.24693300 \\
\hline $\mathrm{H}$ & 13.23474800 & 4.24343900 & -0.25453800 \\
\hline $\mathrm{C}$ & -2.66417400 & -0.46681400 & 0.06663800 \\
\hline $\mathrm{C}$ & -3.09246300 & 0.77920000 & 0.49369200 \\
\hline $\mathrm{C}$ & -3.57910900 & -1.43614000 & -0.31451600 \\
\hline $\mathrm{C}$ & -4.44498800 & 1.05963200 & 0.53445400 \\
\hline $\mathrm{H}$ & -2.35851600 & 1.51920900 & 0.78270500 \\
\hline $\mathrm{C}$ & -4.92808200 & -1.13814600 & -0.27412600 \\
\hline $\mathrm{H}$ & -3.23925900 & -2.40940300 & -0.63434800 \\
\hline $\mathrm{C}$ & -5.39375500 & 0.11052500 & 0.14664000 \\
\hline $\mathrm{H}$ & -4.76961300 & 2.04417200 & 0.84587700 \\
\hline $\mathrm{H}$ & -5.64004000 & -1.90492800 & -0.55174300 \\
\hline $\mathrm{C}$ & -6.83132900 & 0.41356600 & 0.18009700 \\
\hline $\mathrm{C}$ & -7.36194800 & 1.30374000 & 1.12021600 \\
\hline $\mathrm{C}$ & -7.71377400 & -0.17813900 & -0.72947300 \\
\hline $\mathrm{C}$ & -8.71120100 & 1.58855300 & 1.14965300 \\
\hline $\mathrm{H}$ & -6.70597600 & 1.75680400 & 1.85273000 \\
\hline $\mathrm{C}$ & -9.06518200 & 0.10408200 & -0.70381200 \\
\hline $\mathrm{H}$ & -7.32586300 & -0.84952100 & -1.48496300 \\
\hline $\mathrm{C}$ & -9.58005800 & 0.99248000 & 0.23821500 \\
\hline $\mathrm{H}$ & -9.12256100 & 2.27033900 & 1.88259700 \\
\hline $\mathrm{H}$ & -9.73105200 & -0.35551700 & -1.42091700 \\
\hline $\mathrm{C}$ & -11.01378300 & 1.33524100 & 0.31676200 \\
\hline $\mathrm{O}$ & -11.49768500 & 2.09843500 & 1.11710700 \\
\hline $\mathrm{O}$ & -11.74326400 & 0.69202600 & -0.62034600 \\
\hline $\mathrm{C}$ & -13.13627200 & 0.98804500 & -0.59072600 \\
\hline $\mathrm{H}$ & -13.30996500 & 2.05085800 & -0.75373200 \\
\hline $\mathrm{H}$ & -13.57061800 & 0.71228900 & 0.36926800 \\
\hline $\mathrm{H}$ & -13.58520400 & 0.40579900 & -1.38907400 \\
\hline
\end{tabular}

\section{References}

[1] Zhao Y, Truhlar DG. A new local density functional for main-group thermochemistry, transition and metal bonding, thermochemical kinetics, and noncovalent interactions. $\mathbf{J}$ Chem Phys. 2006;125:19410(1-18).

[2] Weigend F, Ahlrichs R. Balanced basis sets of split valence, triple zeta valence and quadruple zeta valence quality for $\mathrm{H}$ to $\mathrm{Rn}$ : Design and assessment of accuracy. Phys Chem Chem Phys. 2005;7:3297-3305. 
[3] Becke AD. Density-functional thermochemistry. III. The role of exact exchange. J Chem Phys. 1993;98:5648-5652.

[4] Krishnan SAR, Weisflog W, Pelzl G, Diele S, Kresse H, Vakhovskaya Z, Friedemann R. DFT and MD studies on the influence of the orientation of ester linkage groups in banana-shaped mesogens. Phys Chem Chem Phys. 2006;8:1170-1177. 